\title{
Diagnosis of Middle East Respiratory Syndrome Coronavirus in Japan
}

\author{
Shutoku MATSUYAMA*
}

Laboratory-4, Department of Virology III, National Institute of Infectious Diseases (NIID)

\begin{abstract}
Cases of pneumonia caused by Middle East respiratory syndrome coronavirus (MERS-CoV) continue to be reported from the Arabian Peninsula, and the adaptation of MERS-CoV to allow more efficient human-to-human transmission is a public health concern. From March 2012 to April 2016, 1,714 cases including 681 deaths of MERS-CoV infection have been identified in 26 countries. Especially, we learned this virus carries potential risk to spread quickly in hospital from the MERS outbreak in South Korea 2015. This virus seems to be more deadly and less contagious than severe acute respiratory syndrome coronavirus (SARS-CoV) in 2003. The MERS-CoV belongs to the lineage $\mathrm{C}$ of the beta coronaviruses, which are genetically and serologically same to a coronavirus detected in dromedary camels lived in the Middle East and Africa. Camel-to-human and human-to-human transmission of pathogen has often been observed in the Middle East.

On alert for a possible spread of virus in Japan, we deployed the real-time RT-PCR targeting MERS-CoV reported by Germany group (Corman VM 2012 Euro Surveill) to 84 of prefectural public health institutes and quarantines in Japan. Additionally, we developed a real-time direct reverse transcription Loop-mediated isothermal amplification (RT-LAMP) assay for detecting MERSCoV. An RT-LAMP assay is simple, rapid, highly sensitive and specific nucleic acid amplification method and more cost effective than the PCR assay. It has been used for diagnosis including point-of-care testing for many pathogens during the past $\sim 15$ years. Our realtime direct RT-LAMP assay has several advantages: 1) no longer requires nucleic acid purification process, making the diagnostic test more rapid and simpler; 2) highly sensitivity and specificity through use of a fluorescence primer; 3) time to detection of positive result is shorter within 20 minutes from collected specimens; and 4) 2 types (A and B) and 4 subtypes (H1pdm09, H3, H7 and H5) of influenza viruses are simultaneously detectable. Another remarkable point of our MERS diagnosis system is unnecessity of RNA extraction step and reaction pre-mixtures making step. The specimen is collected in the Extraction reagent and the reagent is put into the lyophilized RT-LAMP reagents in 8-well strip tubes after filtration. All steps of preparation for RT-LAMP reaction are taken within 2 minutes. Moreover, we have successfully created ready-to-use kit with dried reagents (primers, enzymes and buffer) in one tube. Since the kit is stable in a room temperature, the handling of the kit becomes simple without cooling. RT-LAMP is an isothermal nucleic acid amplification method and will be undergone at a constant temperature of $65^{\circ} \mathrm{C}$ in around 20 minutes. This reaction can be performed using the portable incubator with fluorometer. This simple, rapid and highly sensitive and specific assay is useful for diagnosis of MERS-CoV. The RT-LAMP assay might be a powerful tool for diagnosis in hospital.

Using the assays described above, we test the specimens from dromedary camels to understand the risk of MERS-CoV infection in Japan. A total of 18 fecal specimens, 10 saliva specimens, 4 nasal swabs, and 5 serum specimens were obtained from 20 dromedaries, which is representative of almost $87 \%$ of the dromedaries in Japan. In result, all the specimens were negative for viral RNA by the MERS-CoV specific assays (real-time RT-PCR and RT-LAMP).
\end{abstract}

\section{REFERENCE}

(1) Notomi T, Okayama H, Masubuchi H, Yonekawa T, Watanabe K, Amino N, Hase T., Loop-mediated isothermal amplification of DNA. Nucleic Acid Res. 2000. 15; 28 (12): E63.

(2) Nakauchi M, Yoshikawa T, Nakai H, Sugata K, Yoshikawa A, Asano Y, Ihira M, Tashiro M, Kageyama T., Evaluation of reverse transcription loop-mediated isothermal amplification assays for rapid diagnosis of pandemic influenza A/H1N1 2009 virus. J Med Virol. 2011. 83(1): 10-5.

(3) Shirato K, Yano T, Senba S, Akachi S, Kobayashi T, Nishinaka T, Notomi T, Matsuyama S. Detection of Middle East respiratory

* Corresponding to: Shutoku MATSUYAMA*

4-7-1 Gakuen, Musashi-Murayama, Tokyo 208-0011, Japan

Tel: +81-42-561-0771; Fax: +81-42-567-5631

E-mail: matuyama@nih.go.jp 
syndrome coronavirus using reverse transcription loop-mediated isothermal amplification (RT-LAMP). Virol J. 2014 Aug 8; 11: 139.

(4) Shirato K, Azumano A, Nakao T, Hagihara D, Ishida M, Tamai K, Yamazaki K, Kawase M, Okamoto Y, Kawakami S, Okada N, Fukushima K, Nakajima K, Matsuyama S., Middle East respiratory syndrome coronavirus infection not found in camels in Japan. Jpn J Infect Dis. 2015; 68(3): 256-8. 